\title{
TURBULENT FLOW CONTROL WITH VORTEX GENERATORS AROUND A SYMMETRICAL AIRFOIL AT HIGH ATTACK ANGLES
}

\author{
Hocine Tebbiche ${ }^{1}$, Mohammed Saîd Boutoudj ${ }^{2 *}$ \\ 1 Laboratoire d'Energétique, Mécanique et Matériaux -LEMM; Université Mouloud Mammeri, \\ Tizi-Ouzou, Algeria \\ e-mail: tebbichehocine@yahoo.fr \\ 2 Laboratoire d'Energétique, Mécanique et Matériaux -LEMM; Université Mouloud Mammeri, \\ Tizi-Ouzou, Algeria \\ e-mail: boutoudj_ms@yahoo.fr \\ *corresponding author
}

\begin{abstract}
The flow on the upper surface of NACA 0015 airfoil is subject to an adverse pressure gradient when the incidence increases. This leads to the boundary layer separation which causes losses in the aerodynamic performances. Control using Vortex Generators (VGs) is a simple passive mean used to delay or eliminate the flow separation from the wall.

The two main objectives of the experimental work performed consist in the optimization by experimental design method "DoE" of a new VGs configuration, inspired by Lin's one, by varying its different geometrical parameters. A comparative efficiency study of the two VGs configurations (Lin's VGs, modified VGs) on aerodynamic performances was then undertaken at different Reynolds numbers.

In addition to the significant improvement in lift around $22 \%$, the study resulted in a drag reduction of approximately $16 \%$ and a stall delay of two degrees. The results were also compared to a three-dimensional numerical simulation (3D-RANS) and showed good agreement. The CFD results highlighted a clear improvement in the momentum thickness along the airfoil's upper face, particularly a rate of $44.44 \%$ at $40 \%$ of the chord length.
\end{abstract}

Keywords: Vortex Generators, NACA 0015 airfoil, lift and drag, pressure, design of Experiments, CFD. 


\begin{tabular}{|c|c|c|}
\hline \multicolumn{3}{|c|}{ Nomenclature: } \\
\hline$a$ & : Space between the same VGs (m) & $X, Y, Z$ : Cartesian coordinates (m) \\
\hline$b$ & Space between two VGs (m) & \multirow{2}{*}{$\begin{array}{l}X^{t}: \text { Transposed matrix of the effects } \\
\text { calculation }\end{array}$} \\
\hline$c$ & : Additional element of VGs (m) & \\
\hline$C_{d}$ & : Drag coefficient & : Normal distance to the protile \\
\hline$\overline{C_{f}}$ & : Mean skin friction coefficient & $Y \quad:$ Response-vector \\
\hline$C_{L}$ & : Lift coefficient & $y^{+} \quad$ : Dimensionless distance to the \\
\hline$C_{L \max }$ & : Maximum lift coefficient & wall \\
\hline$C p$ & : Pressure coefficient & Greek symbols \\
\hline $\begin{array}{l}E \\
F_{x}\end{array}$ & $\begin{array}{l}\text { : Effect-vector } \\
: \text { Drag force }(\mathrm{N})\end{array}$ & $\alpha \quad$ : Angle of attack $\left({ }^{\circ}\right)$ \\
\hline$F_{y}$ & : Lift force $(\mathrm{N})$ & : Boundary layer thickness (m) \\
\hline$h$ & : Height of VGs (m) & : Displacement thickness (m) \\
\hline$h_{k}$ & Kline factor & : Momentum thickness (m) \\
\hline $\mathrm{H}_{12}$ & : Shape factor & : Aperture angle of VGs $\left({ }^{\circ}\right)$ \\
\hline$k$ & : Number of factors & : Kinematic viscosity $\left(\mathrm{m}^{2} \cdot \mathrm{s}^{-1}\right)$ \\
\hline$l$ & : Length of VGs (m) & : Density $\left(\mathrm{kg} \cdot \mathrm{m}^{-3}\right)$ \\
\hline $\mathrm{L}$ & : Chord length (m) & \\
\hline$N$ & : Number of experiments & Abbreviations \\
\hline$P$ & : Wall static pressure $(\mathrm{Pa})$ & : 3 Dimensions \\
\hline$P_{0}$ & : Upstream reference pressure $(\mathrm{Pa})$ & : Computational Fluid Dynamics \\
\hline $\operatorname{Re}_{L}$ & : Reynolds number related to the chord length & : Design of Experiments \\
\hline$S$ & : Surface profile $\left(\mathrm{m}^{2}\right)$ & : Experimental data \\
\hline $\begin{array}{l}u \\
\text { surface }\end{array}$ & : Velocity component tangential to the & $\begin{array}{l}\text { NACA: National Advisory Committee for } \\
\text { Aeronautics }\end{array}$ \\
\hline & $(\mathrm{m} / \mathrm{s})$ & RANS : Reynolds Averaged Navier Stokes \\
\hline$U_{\infty}$ & : Freestream velocity $(\mathrm{m} / \mathrm{s})$ & SST : Shear Stress Transport \\
\hline$U_{e}$ & : Maximum tangential speed $(\mathrm{m} / \mathrm{s})$ & VGs : Vortex Generators \\
\hline
\end{tabular}

\section{Introduction}

The work done by Prandtl (1904) has allowed a prompt progress in the aeronautical field by showing the possibility to analyze low viscosity flows such as air around profiles in two zones: a so-called external zone, where the viscosity effects are negligible; the flow in this case is considered potential, and a very thin inner zone in the immediate vicinity of the wall, place of important energy exchanges, called boundary layer (Schlichting. 1979) where the viscosity effects are preponderant. The boundary layer detachment is extremely penalizing and leads to significant losses in terms of aircraft aerodynamic performance and the generation of vibrations and noise. This leads to the need to apply flow control (Gad-el-Hak. 2001).

Control strategies divide into two broad families that may be passive or active. Their principle consists of manipulating the separated flows by various means: adding a momentum to the fluid by suction (Schlichting. 1979) or blowing (Favier et al. 2006), moving surfaces (Steele et al. 1970), influencing the laminar-turbulent transition (Walsh. 1983) or by implantation of specific geometric shapes, namely Vortex Generators (VGs) (Bak et al. 2002 and Lin. 2002).

All of these techniques tend to delay or even eliminate the fluid separation from the wall (Brown et al. 1968), increase the lift and reduce drag (Tebbiche et al. 2015), optimize energy exchanges, improve comfort and reduce noise.

Since the introduction of passive control through vortex generators by Taylor (1947), this technique has been widely used to delay boundary layer separation through parametric studies 
(Lin. 1999, Godard et al. 2006, Zhen et al. 2011, Fouatih et al. 2016 and Hares et al. 2019), to improve the wings lift (Nickerson. 1986), avoid or delay separation in subsonic diffusers (Brown et al. 1968) and reduce aircraft fuselage drag (Calarese et al. 1985). One of the most important VGs geometrical aspects is their height $h$ in relation to the boundary layer thickness $\delta$. Initially, Taylor evaluates this height in the neighborhood of $\delta(h / \delta \approx 1)$. However, Taylor's vortex generators generate significant parasitic drag that results in reduced efficiency.

Two variants of micro-generators were tested by Lin (1999) in delta and trapezoidal wing shapes that can be either co-rotating (CoR) or counter-rotating (CtR). A first conclusion of this work is that the vortices rotating in opposite directions allow a better mixing between the fluid outside the boundary layer and that of low speed, in the wall vicinity. A second Lin's conclusion relates to the relative height of VGs. Thus, a significant improvement in control is obtained for a threshold height of $h / \delta \approx 0.2$. For $h / \delta>0.2$, an increase in the drag is observed without providing a significant improvement in lift, while values of $h / \delta<0.2$ are accompanied by a decrease in the VGs efficiency. Several investigations followed one another, with the main objective being the optimization of geometric parameters, especially the height of VGs (Lin. 2002 and Godard et al. 2006).

Regarding the aspect related to the rotation direction of the vortices, the study conducted by Godard et al (2006) has confirmed Lin's conclusion by highlighting a level of performance greater than $100 \%$ in the case of counter-rotating vortices. Moreover, the optimal height of VGs is evaluated in this work at $37 \%$ of the boundary layer thickness.

As part of this paper, an optimization work of (CtR-VGs) was performed. The basic configuration initially considered is that of Lin (1999), which was modified in order to improve the NACA 0015 profile aerodynamic coefficients. A program of the aerodynamic forces measurements was undertaken by means of a Design of Experiments strategy "DoE" (Montgomery 1991) in order to obtain an optimized configuration of the proposed Vortex Generators.

Increasing number of authors is interested in the use of these experimental designs in order to perform their tests in various areas. We can cite non-exhaustively the research work done by Zeng et al. (2010) who analyzed the influence of various parameters on the heat transfer and flow friction characteristics of a heat exchanger with Vortex Generators fins by numerical method using experimental design. The parameters of vortex generator fin-and-tube heat exchangers were optimized using the Taguchi method (Taguchi. 1993). Again, Lundstedt et al. (1998) present a tutorial which aims to give a simple and easily understandable introduction to experimental design and optimization. The screening methods described in their paper are factorial and fractional factorial designs. This has been carried out in an efficient way and without having to perform a large number of experiments.

All these diverse investigations carried out on different domains using DoE methodology prompt us to present a paper to provide optimized geometry for vortex generators with contrarotative vortices using a full factorial design based on the main shape already used by other authors in particular those reported by Lin (1999). Various velocities of the flow were tested in wind tunnel in order to determine the Reynolds number effect on the control parameters. The results are analyzed in several parameters such as the VG height, the aperture angle, the space between the same VG pair and the additional factor effect.

A comparative study is also made between the proposed optimal vortex generators geometry and the same one without the additional element.

The experimental results remain insufficient to the understanding of the mixture genesis and transporting momentum from outer regions highly energetic to the near-wall subjected to a strong adverse pressure gradient. Indeed, a 3D-RANS numerical simulation campaign with k $₫$-SST 
turbulence model using the commercial code Fluent ${ }^{\mathrm{TM}}$ (Fluent. 2005) completes the experimental study in quantitative and qualitative analysis of the coherent structures generated by VGs.

\section{Experimental setup}

The experiments were performed in a wind tunnel equipped with two measurement systems: pressure field and aerodynamic forces.

\subsection{Wind tunnel and acquisition system}

The studied profile is NACA 0015; the chord length is $150 \mathrm{~mm}$ with a wingspan of $200 \mathrm{~mm}$. It is also equipped with fourteen pressure taps on the upper surface for the pressure field measurement. The lift and drag forces were measured using a strain gauge aerodynamic balance connected to an acquisition chain. Each test performed was repeated three times then the average was considered. The acquisition time was $60 \mathrm{~s}$ with a frequency of $500 \mathrm{~Hz}$. All the experiments were achieved in a Deltalab ${ }^{\mathrm{TM}}$ type wind tunnel. The maximum measurable velocity is greater than $40 \mathrm{~m} / \mathrm{s}$. The turbulence rate was set by a grid at the inlet of $5 \times 5 \mathrm{~mm}^{2}$. The length and cross section of the wind tunnel were respectively $100 \mathrm{~cm}$ and $30 \times 30 \mathrm{~cm}^{2}$. All the components of the wind tunnel as well as the acquisition chain are shown in Figure 1.

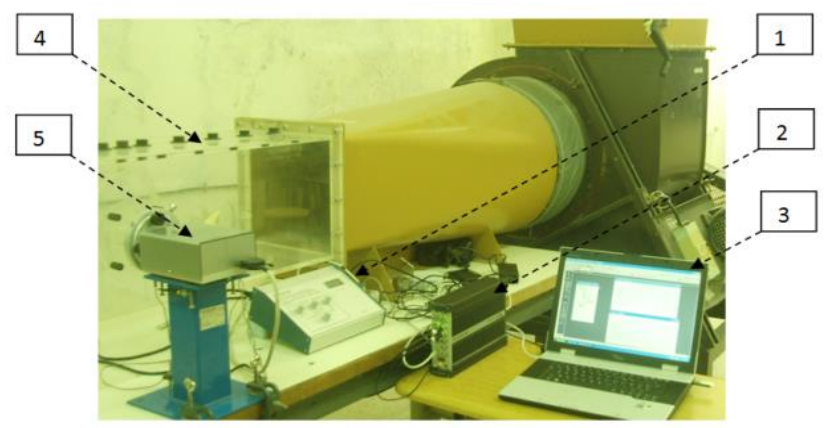

Fig. 1. Measurement setup (1: conditioner, 2: converter, 3: acquisition and processing of data on computer, 4: wind tunnel, 5: aerodynamic balance).

\subsection{Vortex Generators}

Passive control by VGs is a strategy that does not require any external energy to the flow. The implantation of VGs line on the upper face of an aerodynamic profile makes it possible to delay or even eliminate the detachment. Their particularity is to bring the momentum from the external flow to the near-wall flow regions.

Except the additional element ' $c$ ' shown in Figure 2, the vortex generators configuration studied in this paper is the same as that presented by Lin (1999).

Figure 2 shows these VGs while Table 4 summarizes their geometric characteristics retained at the end of the optimization study performed in this paper by design of experiments method. 


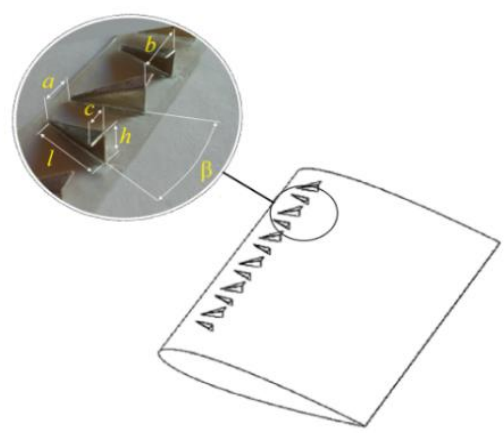

Fig. 2. Passive VGs parameters ( $l$ : vortex generators length, $b$ : distance between two passive devices, $a$ : space between the same $\mathrm{VG}, h$ : vortex generator height, $c$ : vortex generator additional element, $\beta$ : aperture angle).

\section{Global Settings}

The use of shape factor $\left(\mathrm{H}_{12}\right)$ informs us about the state of the boundary layer. It allows the determination of the turbulent laminar transition as well as precise positioning from the location of turbulent boundary layer separation; its expression is given by:

$$
\mathrm{H}_{12}=\frac{\delta_{1}}{\delta_{2}}=\int_{0}^{\infty}\left(1-\frac{u}{U_{\infty}}\right) \mathrm{d} y / \int_{0}^{\infty} \frac{u}{U_{\infty}}\left(1-\frac{u}{U_{\infty}}\right) \mathrm{d} y
$$

These quantities $\left(\delta_{1}\right.$ and $\left.\delta_{2}\right)$, were determined by integration up to the tangential speed maximum value $\left(U_{e}\right)$ of the calculated profile (Thwaites 1987).

The dimensionless coordinate normal to the airfoil $y^{+}$is similar to local Reynolds number, often used in CFD to describe how coarse or fine a mesh is for a particular flow. The nondimensional wall parameter is defined as:

$$
y^{+}=\frac{y U_{\infty} \sqrt{\overline{C_{f}} / 2}}{v}
$$

By assimilating the airfoil to a flat plate, the skin friction coefficient can be estimated from the following empiric relation (Gerasimov. 2006):

$$
\bar{C}_{f} / 2 \approx 0.037 \operatorname{Re}_{L}^{-0.2}
$$
by:

The measured forces (lift and drag) are respectively linked to the aerodynamics coefficients

$$
C_{L, d}=\frac{F_{x, y}}{1 / 2 \rho U_{\infty}^{2} S}
$$

The pressure coefficient $C p$ is provided by the expression:

$$
C p=\frac{P-P_{0}}{1 / 2 \rho U_{\infty}^{2}}
$$




\section{Organization of tests by experimental designs}

An optimization method by experimental designs was used in this study. The optimization procedure of the vortex generators parameters is detailed in the following sections.

\subsection{Formalization of the problem}

The need for employing a rational step (Sado et al. 2000) to carry out research has encouraged the engineers and researchers to employ the statistical methods. The experimental designs have for main goal obtaining the maximum information at lower cost. The desired information is in general to qualify the influence of several parameters (or factors) on a given phenomenon. Based on this information, it will be possible to determine the behavior of the studied system in the various possible configurations, and thus to optimize the answer. To reach this result, the experimental designs technique proposes a strategy of tests having a principal characteristic to minimize the tests number to be realized (Montgomery 1991).

This DoE method is therefore used in this paper to optimize the VGs configuration previously presented in Figure 2. Only four elements $(a, h, c, \beta)$ related to the VG's geometry are used. The other parameters such as the ratios $l / h$ and $b / c$ are maintained constant $(l / h=2.6, b / c=3)$. Level of each factor is shown in Table 1, where level 1 and level 2 represents respectively the low and high values.

\begin{tabular}{l|l|l|l|l}
\hline Code & Factor & Level 1 & Level 2 & Units \\
\hline $\mathrm{A}$ & $a / \delta$ & 0.55 & 0.70 & - \\
\hline $\mathrm{B}$ & $c / \delta$ & 0.30 & 0.45 & - \\
\hline $\mathrm{C}$ & $h / \delta$ & 0.35 & 0.55 & - \\
\hline $\mathrm{D}$ & $\beta$ & 30 & 48 & $\left(^{\circ}\right)$ \\
\hline
\end{tabular}

Table 1. Variation level on each factor

The factors constituting the VGs geometry are expressed in dimensionless form with respect to the boundary layer thickness $\delta$ (see Table. 2). The state of the incompressible turbulent flow around the NACA 0015 profile is solved numerically using (2D-RANS) equations through the finite volume method, by means of the Fluent ${ }^{\mathrm{TM}}$ calculation code. The turbulence model chosen is $\mathrm{k} \omega$-SST, keeping the size of the wall parameter at $\mathrm{y}^{+} \approx 1$. The flow Reynolds number is brought to $2.610^{5}$.

The characteristics of the boundary layer at $\mathrm{X} / \mathrm{L}=18.7 \%$ from the leading edge are summarized in Table 2. The overlaying, with experimental measurements, of the pressure coefficient evolutions along the NACA 0015 profile carries out the validation of the CFD results; the results show a good correlation (see Fig. 3).

\begin{tabular}{l|l|l|l|l|l}
\hline $\mathrm{X} / \mathrm{L}$ & $U e$ & $\delta$ & $\delta_{1} \times 10^{4}$ & $\delta_{2} \times 10^{4}$ & $\mathrm{H}_{12}$ \\
\hline 0.187 & 24.84 & 0.01 & 9.91 & 5.08 & 1.95 \\
\hline
\end{tabular}

Table 2. Boundary layer characteristics, $\alpha=13^{\circ}$, CFD. 


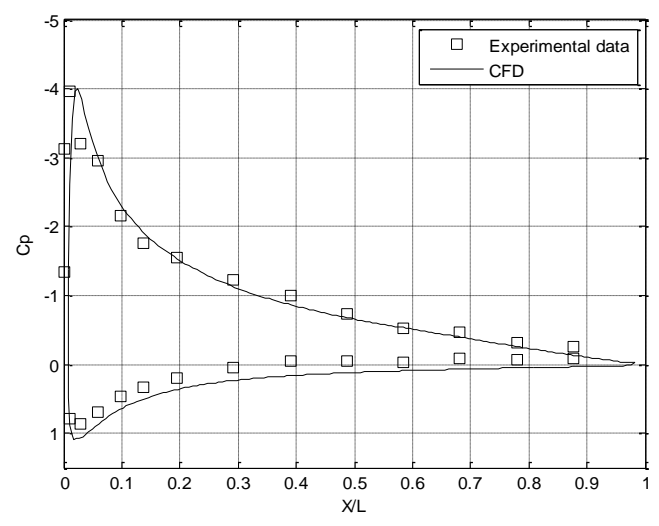

Fig. 3. Pressure coefficient distribution versus $X / L, \alpha=13^{\circ}$.

\subsection{Experimental design selection}

Using a full factorial design with four factors $k$ and two variation levels justifies making sixteen experiments $\left(2^{k}=16\right)$. In the framework of this comparative study, we limit the number of VGs pairs to six. The lift coefficient was selected as objective function (Level 1:-1, Level 2:+1). Table 3 shows the experiences organization and the factor levels for each test.

\begin{tabular}{|c|c|c|c|c|c|c|c|c|c|c|c|c|c|c|c|c|}
\hline \multirow{2}{*}{ Variables } & \multicolumn{16}{|c|}{ Exp. no. } \\
\hline & 01 & 02 & 03 & 04 & 05 & 06 & 07 & 08 & 09 & 10 & 11 & 12 & 13 & 14 & 15 & 16 \\
\hline $\mathrm{A}$ & -1 & +1 & -1 & +1 & -1 & +1 & -1 & +1 & -1 & +1 & -1 & +1 & -1 & +1 & -1 & +1 \\
\hline B & -1 & -1 & +1 & +1 & -1 & -1 & +1 & +1 & -1 & -1 & +1 & +1 & -1 & -1 & +1 & +1 \\
\hline $\mathrm{C}$ & -1 & -1 & -1 & -1 & +1 & +1 & +1 & +1 & -1 & -1 & -1 & -1 & +1 & +1 & +1 & +1 \\
\hline $\mathrm{D}$ & -1 & -1 & -1 & -1 & -1 & -1 & -1 & -1 & +1 & +1 & +1 & +1 & +1 & +1 & +1 & +1 \\
\hline
\end{tabular}

Table 3. A $2^{4}$ factorial experiment.

\subsection{Tests procedure}

The tests were performed by way of the described devices in Figure 1 at Reynolds number of 2.6 $10^{5}$. The obtained results for the reference state (without VGs) indicate that the airfoil's stall angle is observed at 15 degrees (see Fig. 7.(b)).

The DoE method was applied in the case of a post-stall incidence (16 degrees). The objective is the analysis of the influence on the lift of the different factor's combinations mentioned in Table 3 . Figure 4 shows the results of aerodynamic measurements obtained from the different VG's configurations compared to the uncontrolled case at $16^{\circ}$ of incidence. 


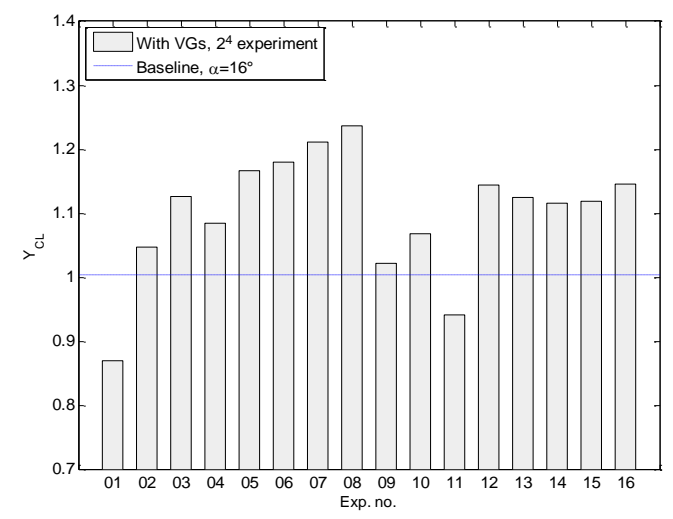

Fig. 4. Lift coefficient at 16 degrees for different sixteen experiments.

\subsection{Analysis of the results}

The effects (interactions) are obtained via the calculation matrix given by this expression (Pareto. 1964):

$$
E=\frac{1}{N} X^{t} Y
$$

Before carrying out the analysis, unnecessary and insignificant factors are eliminated. The presence of insignificant parameters amplifies error and reduces the accuracy and adequacy of the analysis. A classification through the Pareto diagram (Pareto 1964) was generated for this purpose.

The calculation effect of the main factors and interactions is shown in Figure 5. It can be noted that $34.7 \%$ of the causes represent $80 \%$ of the effects. Pareto's law can, in this case, can be used with precaution (Koch 2011). The principle of Pareto's law does not necessarily imply an 80-20 distribution, but that about $80 \%$ of the effects are the product of $20 \%$ of the causes (Joseph 1954).

The factors and interactions that contribute to $80 \%$ of the effects in this study are therefore $(\mathrm{C}, \mathrm{A}$, $\mathrm{B}, \mathrm{ABD}, \mathrm{BD}, \mathrm{ABCD}, \mathrm{CD}, \mathrm{AC}$ and $\mathrm{D})$ for an incidence $\alpha=16^{\circ}$. The classification of the factors effect and interactions through graphical analysis (Fig. 5) highlighted the importance of the Cfactor, represented by the VGs height, which is the most influential factor with a contribution ratio of $22 \%$. This value is calculated on the basis of the $\mathrm{C}$-factor contribution $(\mathrm{E}=0.0625)$ compared to the overall effect. Contrary to the existing literature (Lin. 1999), which informs us that we can naturally expect an increase in drag as the value of $h / \delta$ increases without improvement in lift, the analysis of the previous results (see Fig. 4 and Table. 3) appears clearly that the Cvalues taken on $+1(h / \delta=0.55)$ perform better than those on $-1(h / \delta=0.35)$. As example, the experiment number one $(\mathrm{C}=-1)$ shows less performance than the fifth one $(\mathrm{C}=+1)$ when the other factors (A, B and D) remain unchanged.

Several researchers have investigated the effect of the VGs spacing (Godard et al. 2006 and Ahmad et al. 2005). The single contribution of the factor "A" in the present study is $10 \%$, it is also considered as major element, which justifies the interesting position in the ranking.

The effectiveness of the control through developed VGs is dependent on the VG's height, their spacing, but also the size of the additional element (B-Factor). The addition of this factor to the basic configuration results in a contribution of $9 \%$; this improvement comes mainly from the 
formation of a second pair of counter-rotating vortices (see $\S 6.2 .2$ ). Moreover, a comparative study is devoted to his introduction's effect on the aerodynamic coefficients.

Another equally important finding is covered on D-Factor. Treated alone, it has practically no effect but it may interact with the other factors. Then, the combined contribution (ABD, BD, CD and D) operates on $39 \%$ of the significant effects.

Pareto chart graphical analysis of the effects and interactions involved, proved to be effective in detecting the impact of each factor on the control effectiveness. However, the understanding of the boundary layer reattachment mechanics induced by VGs is necessary. To overcome this insufficiency, a quantitative and qualitative exploration of the flow is carried out experimentally and numerically in the following sections (see $§ 5$ and $\S 6$ ).

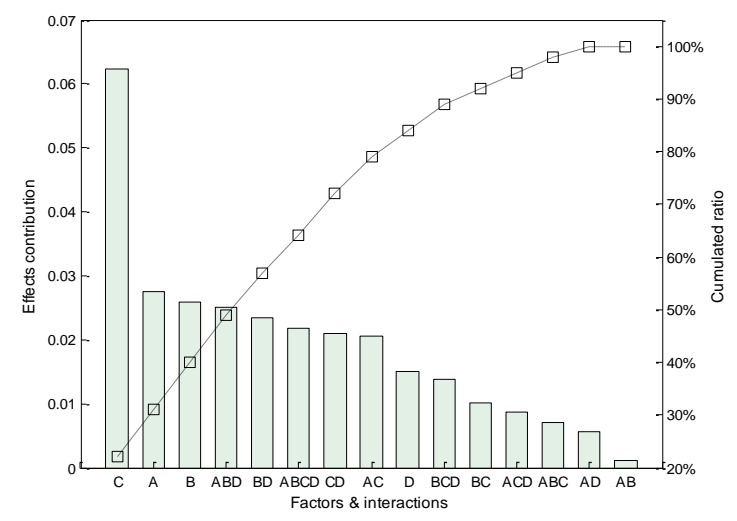

Fig. 5. Pareto diagram applied at 16 degrees, - - $\square-$-cumulated ratio and the bars represents effects contribution.

The results of the optimization study by DoE method allowed us to define a final configuration of four parameters. The optimized geometrical parameters of the Vortex Generators are summarized in Table 4.

\begin{tabular}{l|l|l|l|l}
\hline Factors & $a / \delta$ & $c / \delta$ & $h / \delta$ & $\beta$ \\
\hline Levels & 0.70 & 0.45 & 0.55 & $30^{\circ}$ \\
\hline
\end{tabular}

Table 4. Optimized geometrical parameters.

\section{Experimental results}

This part is an experimental investigation of the flow control around a NACA 0015 profile using the VGs previously defined in Table 4.

\subsection{Position of the vortex generators}

The VGs were positioned in line at 10\% from the leading edge (see Fig. 6); the measurements of the aerodynamic forces were performed for several incidences. When the flow is not controlled, separation is two-dimensional (Mccormick. 2000); only one measurement of the pressure fields is sufficient to obtain the pressure distribution around the profile. On the other hand, when control intervenes, the flow will be three-dimensional. A complete sweeping of span $\Delta \mathrm{Z}$ is necessary and was possible by relocating the $\mathrm{VGs}$ along the $\mathrm{Z}$ axis. 


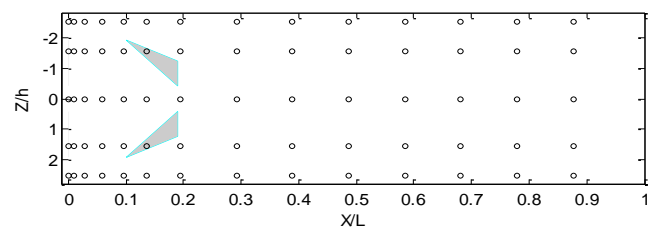

Fig. 6. VGs and pressure taps positions.

\subsection{Lift, drag and pressure measurements}

The experimental results obtained concerning the lift, the drag and the wall pressure field are presented below for controlled and uncontrolled flows.

\subsubsection{Reynolds number effect}

The lift and drag coefficients resulting from the flow around the airfoil without vortex generators versus the incidence angle (uncorrected for wind tunnel blockage) are shown in Figs. 7(a) and 7(b) at two Reynolds numbers. We observe that at low incidence both $C_{L}$ and $C_{d}$ evolutions have a linear behavior. It is also noted that the progressive incidence increase causes a sudden drop in the lift related to a profile stall. This fall is accompanied by an expansion of the induced drag caused by the fluid separation.

Furthermore, stall angles corresponding to Reynolds numbers of $1.5810^{5}$ and $2.610^{5}$ are respectively 13 and 15 degrees. The flow is more resistant to the stall at high Reynolds number.
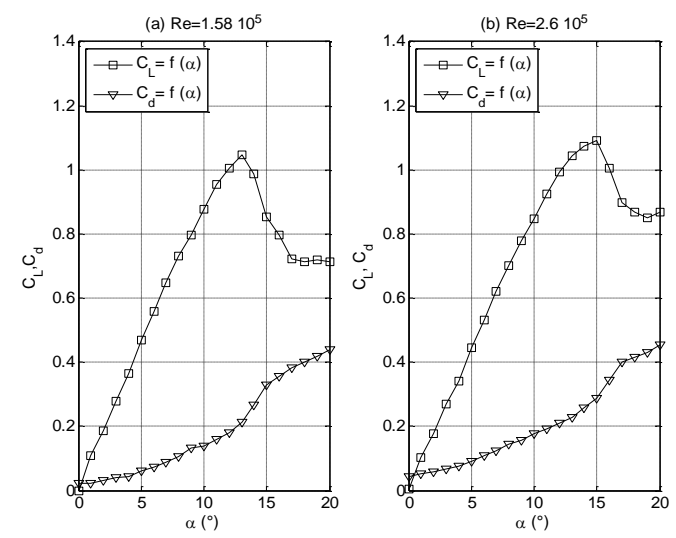

Fig. 7. Lift and drag coefficient versus angle of attack at two Reynolds numbers

\subsubsection{Optimized vortex generators in improvement of the aerodynamic coefficients}

The purpose of this experiment is to demonstrate the VGs ability to change the natural flow on the upper surface of the airfoil. Figure 8 shows the lift coefficient without and with the control geometry given in Table 4. For both speeds studied, lift increase is noticed. At Reynolds number equal to $1.5810^{5}$ (see Fig. 8(a)), the control effect on the lift coefficient is less effective than the case when Reynolds equals $2.610^{5}$ (see Fig. 8(b)). One can see a relative lift increase of $22.2 \%$ in the case (b) and only $5 \%$ for the case (a). The results also show an improvement in the stall angle of two degrees for the two cases. 
The analysis of the drag curves (see Fig. 9) reveals more efficiency of the VGs on the drag reduction at low velocity flow. The drag decrease $\Delta C_{d}$ is about $16 \%$ at low Reynolds number (see Fig. 9(a)) and 2\% for the high speed (see Fig. 9(b)).

However, the $C_{L} / C_{d}$ ratio is increased by $51.3 \%$ for $\mathrm{Re}=2.610^{5}$ and $52.1 \%$ for $\mathrm{Re}=1.5810^{5}$, respectively at 17 and 15 degrees.
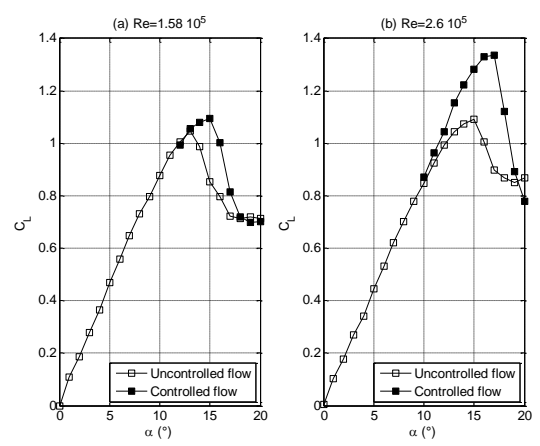

Fig. 8. Lift coefficient versus angle of attack.
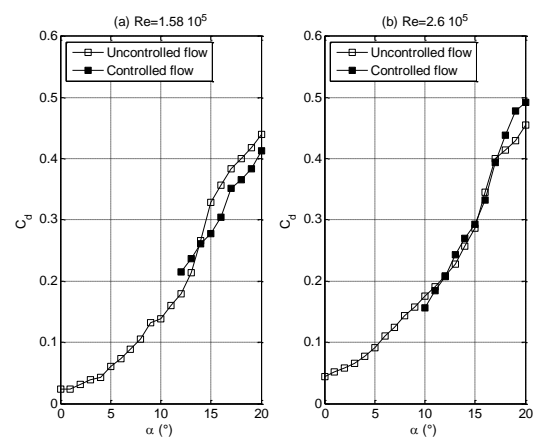

Fig. 9. Drag coefficient versus angle of attack.

\subsubsection{Comparative study of the added B-factor contribution}

A comparative study was made between the proposed VGs geometry and the same one without the factor B in order to determine its influence in the improvement of the aerodynamic performances. About $2 \%$ of lift increase is noticed in Figure 10 when the VGs are equipped with the factor B for the incidences smaller than the stall angle. Figure 11 indicates an increase of about $5 \%$ at the maximum lift. 


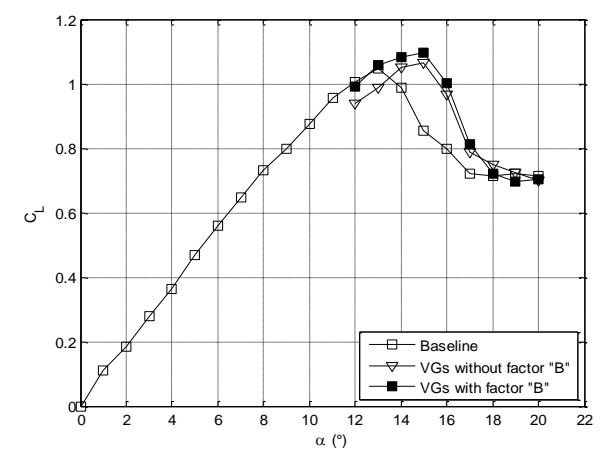

Fig. 10. Lift coefficient versus attack angle, $\operatorname{Re}=1.5810^{5}$.

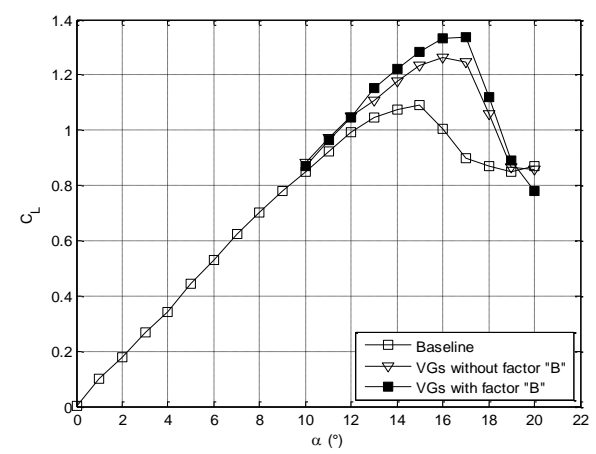

Fig. 11. Lift coefficient versus angle of attack, $\operatorname{Re}=2.610^{5}$.

When the control is applied, the flow becomes three-dimensional, different from the twodimensional one without the VGs. The wall pressure field was investigated in order to study the VGs impact on the pressure evolution.

The following curves show this pressure field on the upper airfoil surface. The measurements were performed at five pressure taps locations along the $\Delta \mathrm{Z}$ space. Curve smoothing was carried out by interpolation to find the intermediates values.

\section{a) Case with factor $B$}

Pressure field outlined in Figures 12(a) and 12(b) shows a periodic distribution of the wall pressure on the upper profile surface. A strong depression is observed in the spacing defined by the factor $\mathrm{A}$. The control highlights the presence of a vortices pair which extends to a very large distance from the leading edge. The flow is not only affected downstream of the vortex generators as shown in the iso-values distribution (see Figs. 13(a) and 13(b)) but also upstream of VGs. The created vortices may thus accelerate the fluid and create a low pressure zone. This energy supply revitalizes the previously separated boundary layer and delays the stall angle. 


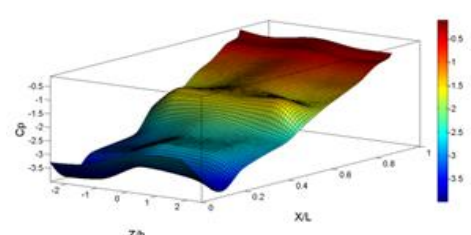

(a) $\operatorname{Re}=1.58 \cdot 10^{5}, \alpha=15^{\circ} \alpha$

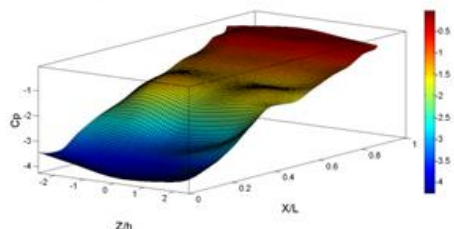

(b) $\cdot \operatorname{Re}=2.6 \cdot 10^{5}, \alpha=16^{\circ} \alpha$

Fig. 12. 3D pressure coefficient, case with factor B.

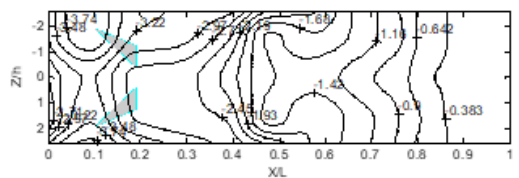

(a) $\operatorname{Re}=1.58 \cdot 10^{5}, \alpha=15^{\circ} \alpha$

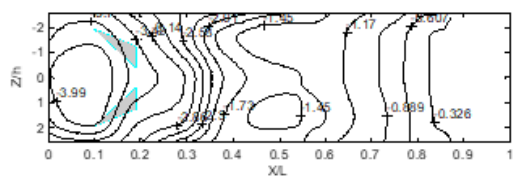

(b) $\cdot \operatorname{Re}=2.6 \cdot 10^{5}, \alpha=16^{\circ} \alpha$

Fig. 13. Iso-values of the pressure field coefficients, case with factor B.

\section{b) Case without factor $B$}

Figures 14 and 15 illustrate the pressure field coefficient at two different velocities and two attack angles in the case without the Factor B. Compared with the optimized shape; we notice an asymmetrical distribution of the pressure field. This can be also seen in the iso-values representations.

On the other hand, the boundary layer reenergized process is more efficient in the presence of the factor B in terms of the pressure field distribution and Lift enhancement (see Figs. 10 and $11)$. 


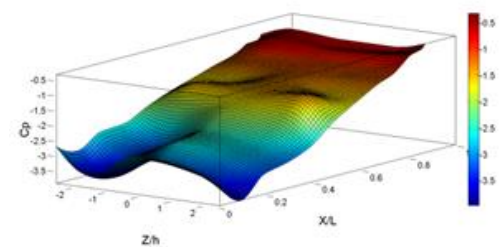

(a) $\cdot \operatorname{Re}=1.58 \cdot 10^{5}, \cdot \alpha=15^{\circ} \alpha$

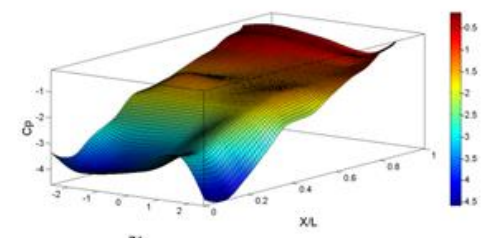

(b) $\operatorname{Re}=2.6 \cdot 10^{5}, \alpha=16^{\circ} \alpha$

Fig. 14. 3D pressure coefficient, case without factor B.

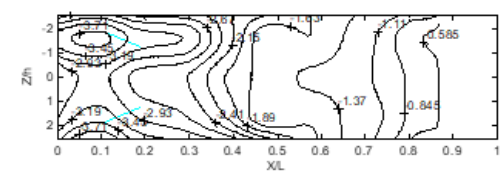

(a) $\operatorname{Re}=1.58 \cdot 10^{5}, \alpha=15^{\circ} \alpha$

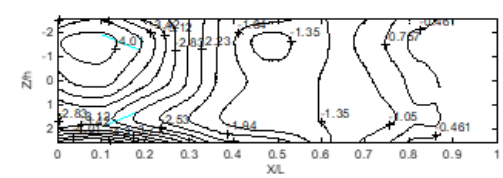

(b) $\cdot \operatorname{Re}=2.6 \cdot 10^{5}, \alpha=16^{\circ} \cdot \alpha$

Fig. 15. Iso-values of the pressure field coefficients, case without factor B.

\section{3D-RANS numerical simulation}

A 3D-RANS numerical simulations campaign is carried out in order to complete the experimental study. A comparison between the CFD and the experimental results is given in the following sections.

\subsection{Methodology}

In order to reduce the computation time and a faster convergence towards the desired solution, it was decided to simulate only a single vortex generator in the previously defined space (see Fig. 6), by adopting the geometrical parameters of the obtained optimal configuration (see Table. 4).

The generated 3D mesh is hybrid; its advantages lie in the fact that it combines the privileges of structured mesh and those of unstructured one by reducing digital errors. Near the airfoil's walls, the generated meshes are hexahedral types. The rest of the calculation box is meshed using prismatic cells (see Fig. 16). As for the VGs, for the reason of the studied complex geometry, they are delimited by a sub-domain and meshed with hexahedral, tetrahedral and pyramidal cells. 

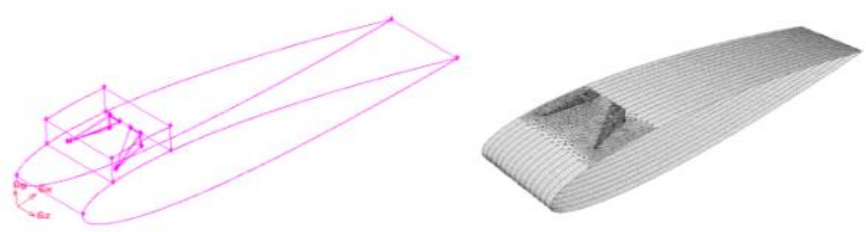

Fig. 16. Different mesh types applied to the calculation box.

\subsection{Results}

The effect of the longitudinal vortices generated by the VGs is studied here through a qualitative and quantitative analysis of the numerical results.

\subsubsection{Validation of the CFD study by lift and drag coefficients}

Figure 17 shows the lift and drag curves resulting from the experimental measurements and those evaluated by the 3D-RANS numerical calculations as function of the attack angle. In terms of lift coefficient, the comparison of the two controlled evolutions case shows a good correlation over the whole range of tested incidences. The CFD study also made it possible to accurately detect the stall angle. In terms of drag coefficient, the numerical values are subject to correction due to a lack of guard-plates. This correction consists to adding a parasite drag to the calculated values.

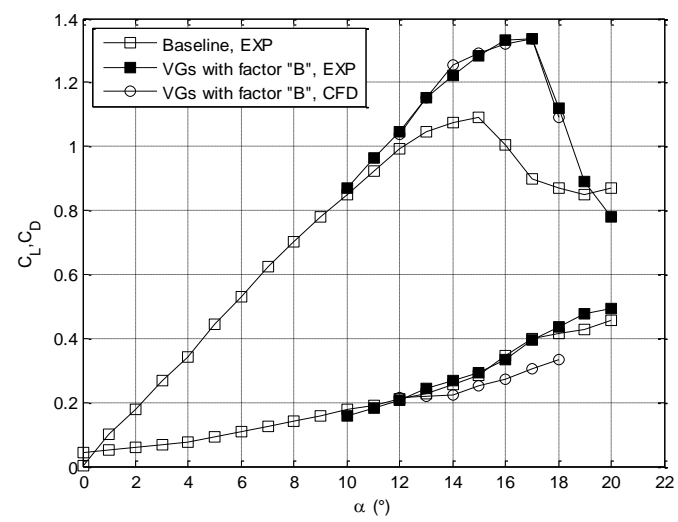

Fig. 17. Lift and drag coefficient versus angle of attack $\alpha$, experimental and numerical data, NACA 0015, $\operatorname{Re}=2.610^{5}$.

\subsubsection{Velocity fields}

The topology of the three-dimensional flow is visualized thanking to the longitudinal Isocontours of mean velocity in the plane $(\mathrm{Y}, \mathrm{Z})$ for three positions according to the chord (see Fig. 18). The studied incidence is fixed at 16 degrees; the sketched maps make it possible to highlight the vortex structures which allow the reattachment of the fluid. The formation of span vortices just downstream of the VG is perfectly noticeable. These well-distinguished vortices supply the boundary layer with a high fluid velocity, occurred from the change in its direction from the fluidVGs interaction. Increasing the flow velocity causes a more pronounced effect. The maps 
extracted at different positions to the chord length show that the rotational vortices create locally an orientation of the flow towards the airfoil's wall.

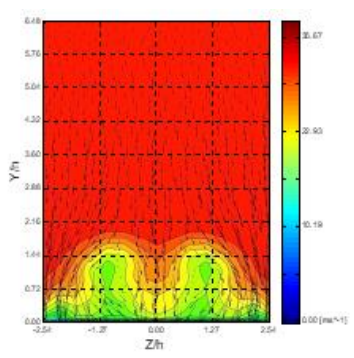

(a) $\mathrm{X} / \mathrm{L}=0.3$

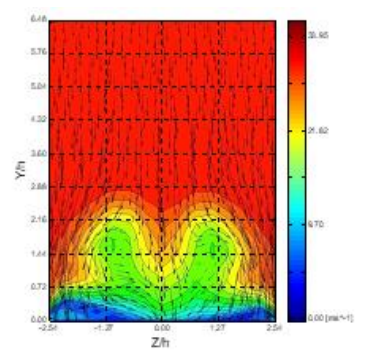

(b) $\mathrm{X} / \mathrm{L}=0.4$

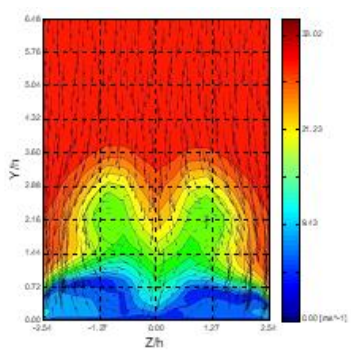

(c) $\mathrm{X} / \mathrm{L}=0.5$

Fig. 18. Field contours of the averaged velocity at different positions to the chord, $\alpha=16^{\circ}$,

$$
\operatorname{Re}=2.610^{5} \text {. }
$$

\subsubsection{VG's Contribution in improvement of the boundary layer parameters}

The detachment of turbulent boundary layers appears from a threshold value of the shape factor $\mathrm{H}_{12}$ equal to 2.3 (Bradshaw. 1969) and which is represented in Figure 19 by dashed horizontal line. This limit is reached for the reference case at around of $\mathrm{X} / \mathrm{L}=0.2$. The evolution of the shape factor increases until reaching $\mathrm{H}_{12}=2.7$ which indicates a state of intermittent detachment of the boundary layer (Kline et al. 1983). The evolutions of the $\mathrm{H}_{12}$ parameter for the controlled case are sketched in solid symbols at two wingspan positions $(\mathrm{Z} / \mathrm{h}=0.00$ and $\mathrm{Z} / \mathrm{h}=1.56)$. At $\mathrm{Z} / h=0.00, \mathrm{H}_{12}$ describes an attached flow to the wall from the leading edge to about $\mathrm{X} / \mathrm{L}=0.6$. From this position, the control efficiency fades, the flow decollate from the wall $\left(\mathrm{H}_{12}>2.3\right)$.

For $\mathrm{Z} / h=1.56$, identified as the interaction zone of the two contrarotative vortices, the flow is reattached to almost all of the profile's upper surface until $\mathrm{X} / \mathrm{L}=0.9$.

Figure 20 shows the superposition of the velocity profiles obtained at $\mathrm{X} / \mathrm{L}=0.4$, while the quantification of the boundary layer variables contribution is summarized in Table 5. A comparison between experimental and numerical results is carried out through the superposition of aerodynamic forces of lift coefficient $C_{L}$; the results show good agreement. Indeed, the maximum relative error recorded is $2.57 \%$ for the uncontrolled case and only $1.41 \%$ for the controlled case.

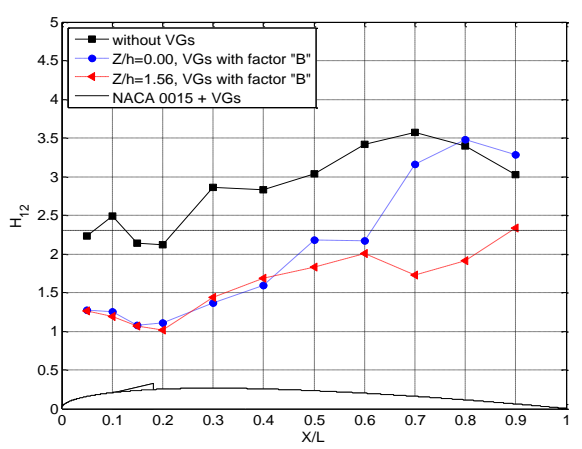

Fig. 19. Evolution of the shape factor in controlled flow case compared to the reference one, $\alpha=16^{\circ}$. 


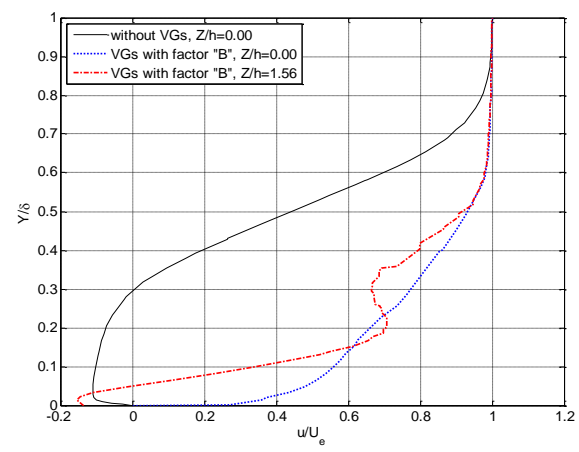

Fig. 20. Comparison of the velocity profiles sketched at $X / L=0.4, \alpha=16^{\circ}$.

The Table 5 shows a significant reduction of the displacement thickness given by $\delta_{l}$. The improvement of $\delta_{2}$, defined as the momentum thickness, of $44.44 \%$ at $\mathrm{Z} / \mathrm{h}=0.00$ and $33.33 \%$ at $\mathrm{Z} / h=1.56$, implies a significant revitalization of the boundary layer. The value of the factor $h_{k}$ $=0.6469$ relative to the uncontrolled case on which incipient detachment was observed seems to be in good agreement with the limit proposed by Kline et al (1983). With the control by VGs, the value of the factor $h_{k}$ drops by $-42.32 \%$ at $\mathrm{Z} / h=0.00$ and by $-37.13 \%$ at $\mathrm{Z} / h=1.56$, indicating that the new vortex generators configuration reduces significantly the adverse effects of backflow.

\begin{tabular}{|c|c|c|c|c|c|c|c|c|c|c|}
\hline & $\mathrm{CL}_{\mathrm{CFD}}$ & $\mathrm{CL}_{\text {Exp }}$ & $\begin{array}{l}\text { Relativ } \\
\text { e error }\end{array}$ & $Z / h$ & Ue & $\delta$ & $\delta_{l}$ & $\delta_{2}$ & $\mathrm{H}_{12}$ & $h_{k}={ }^{\left(\mathrm{H}_{12}-1\right)} / \mathrm{H}_{12}$ \\
\hline No $V G s$ & $\begin{array}{c}0.983 \\
2\end{array}$ & $\begin{array}{c}1.009 \\
1\end{array}$ & $2.57 \%$ & $\begin{array}{c}0.0 \\
0\end{array}$ & $\begin{array}{c}34.211 \\
6\end{array}$ & $\begin{array}{c}0.024 \\
3\end{array}$ & $\begin{array}{c}0.005 \\
2\end{array}$ & $\begin{array}{c}0.001 \\
8\end{array}$ & $\begin{array}{c}2.831 \\
8\end{array}$ & 0.6469 \\
\hline \multirow{2}{*}{$\begin{array}{l}\text { With } \\
\text { VGs }\end{array}$} & \multirow{2}{*}{$\begin{array}{c}1.315 \\
4\end{array}$} & \multirow{2}{*}{$\begin{array}{c}1.334 \\
2\end{array}$} & \multirow{2}{*}{$1.41 \%$} & $\begin{array}{c}0.0 \\
0\end{array}$ & $\begin{array}{c}33.883 \\
8 \\
\end{array}$ & $\begin{array}{c}0.026 \\
5\end{array}$ & $\begin{array}{c}0.004 \\
1\end{array}$ & $\begin{array}{c}0.002 \\
6\end{array}$ & $\begin{array}{c}1.593 \\
0 \\
\end{array}$ & 0.3722 \\
\hline & & & & $\begin{array}{c}1.5 \\
6\end{array}$ & $\begin{array}{c}33.842 \\
9 \\
\end{array}$ & $\begin{array}{c}0.026 \\
5 \\
\end{array}$ & $\begin{array}{c}0.004 \\
0 \\
\end{array}$ & $\begin{array}{c}0.002 \\
4 \\
\end{array}$ & $\begin{array}{c}1.685 \\
4 \\
\end{array}$ & 0.4067 \\
\hline \multirow{2}{*}{$\begin{array}{c}\text { Variatio } \\
n\end{array}$} & & & & $\begin{array}{c}0.0 \\
0 \\
\end{array}$ & $0.95 \%$ & $\begin{array}{c}9.05 \\
\% \\
\end{array}$ & $\begin{array}{c}-21.15 \\
\% \\
\end{array}$ & $\begin{array}{c}44.44 \\
\%\end{array}$ & $\begin{array}{c}-43.75 \\
\% \\
\end{array}$ & $-42.32 \%$ \\
\hline & & & & $\begin{array}{c}1.5 \\
6\end{array}$ & $1.08 \%$ & $\begin{array}{c}9.05 \\
\%\end{array}$ & $\begin{array}{c}-23.08 \\
\% \\
\end{array}$ & $\begin{array}{c}33.33 \\
\%\end{array}$ & $\begin{array}{c}-40.48 \\
\%\end{array}$ & $-37.13 \%$ \\
\hline
\end{tabular}

Table. 5. Global settings, $\alpha=16^{\circ}, \mathrm{X} / \mathrm{L}=0.4$.

\section{Conclusion}

The experimental investigation enabled us to carry out tests relating to the control of aerodynamic unhooking by setting up an optimization step of the vortex generators shape parameters by the mean of the experimental designs. The boundary layer control has shown better aerodynamic performance which results in improved drag and lift, and increased stall angle. The main results obtained by this contribution can be summarized as follows:

- The graphical study underlined the importance of the $\mathrm{C}$-factor, represented by the vortex generators height, which is considered as the most influential factor with a contribution ratio of $22 \%$. The analysis of height effect results revealed an optimal geometry of VGs showing an improvement of $22.2 \%$ relative lift compared to $C_{L} \max$ and $16 \%$ of drag reduction.

- Comparative efficiency of the studied VGs highlighted a significant improvement on the flow control when the vortex generators are equipped with B-factor. This result is 
confirmed by aerodynamic weighing, the three-dimensional representation of the pressure field as well as the iso-values curves.

- The tridimensional pressure field plots of the two studied configurations showed interesting results. Indeed, concerning all the forms tested, it was found that the $C p$ fields are affected downstream as well as upstream of vortex generators line implanted on the upper face of the NACA 0015 airfoil. A strong peak of depression reaching about $C p \approx$ -4 is observed just at the profile's leading edge for both VG's tested which increases to about $35 \%$ of the chord. From this threshold, the evolutions of $3 \mathrm{D}-\mathrm{Cp}$ find a quasiconstant level.

The Reynolds number effect was also performed; it shows that the flow at high velocity is more effective in increasing the Lift/Drag ratio.

The experimental results obtained were completed by a three-dimensional numerical approach expressed by the qualitative and quantitative exploration of the vortex structures responsible for the reattachment of the fluid. A good correlation between the two approaches has been demonstrated by the superposition of aerodynamic force curves. The qualitative aspect of vortex structures revealed the counter-rotating nature of the vortices.

Quantitative analysis of the effects of boundary layer control has shown the effectiveness of vortex structures in reattaching the separated flow from the wall. The evaluation of this contribution is noticeable by comparing the boundary layer parameters of both controlled and uncontrolled cases. Indeed, in the case of the NACA 0015 airfoil, the control by the VGs equipped with factor "B" allows a clear shape factor reduction along the chord, particularly a rate of $-43.75 \%$ at $\mathrm{X} / \mathrm{L}=40 \%$ of the chord on the middle line $(\mathrm{Z} / \mathrm{h}=0.00)$.

The proposed passive vortex generators are simple devices to control the separated flow around an airfoil by bringing momentum in the boundary layer, which leads to maintaining the flow attached to the wall. Their interest is all the greater as they also have the advantage to be retractable when not in use. It is therefore interesting to complete this work with velocity field's measurement and to carry out an unsteady analysis by experimental and numerical studies of the reattachment mechanics induced by the vortex generators.

\section{References}

Ahmad K A, Watterson J K, Cole J S, Briggs I (2005). Sub-boundary layer vortex generator control of a separated diffuser flow. 35th AIAA Fluid Dyn Conf Exhib, 4650.

Bak C, Fuglsang P, Johansen J, Antoniou I (2002). Wind Tunnel Tests of the NACA 63-415 and a Modified NACA63-415 Airfoil. Fors $\phi$ gsanlag Ris $\phi$.

Bradshaw P (1969). The response of a constant pressure turbulent boundary layer to the sudden application of an adverse pressure gradient. HM Stationery Office.

Brown A C, Nawrocki H F, Paley P N (1968). Subsonic diffusers designed integrally with vortex generators. J Aircr, 5: 221-229.

Calarese W, Crisler W, Gustafson G (1985). Afterbody drag reduction by vortex generators. Aerospace Sciences Meetings, American Institute of Aeronautics and Astronautics.

Favier J, Kourta A (2006). Étude du contrôle du décollement sur un profil d'aile par mesures PIV et analyse POD. Comptes Rendus-Mec, 334:272-278.

Fluent (2005). Fluent 6.2 User's Guide Fluent inc. NH, USA.

Fouatih O M, Medale M, Imine O, Imine B (2016). Design optimization of the aerodynamic passive flow control on NACA 4415 airfoil using vortex generators. Eur J Mech B/Fluids, 56:82-96.

Gad-el-Hak M (2001). Flow Control: The Future. J Aircr, 38:402-418. 
Gerasimov A (2006). Modeling Turbulent Flows With FLUENT. Ansys Inc, Sheffield.

Godard G, Stanislas M (2006). Control of a decelerating boundary layer. Part 1: Optimization of passive vortex generators. Aerosp Sci Technol, 10:181-191.

Hares H, Mebarki G, Brioua M, Naoun M (2019). Aerodynamic performances improvement of NACA 4415 profile by passive flow control using vortex generators. Journal of the Serbian Society for Computational Mechanics, 13(1):17-38.

Joseph M J (1954). Universals in management planning and controlling. Management Review, 43:748-761.

Kline S J, Strawn R C, Bardina J G (1983). Correlation of the detachment of two-dimensional turbulent boundary layers. AIAA J, 21:68-73.

Koch R (2011). The 80/20 Principle: The secret of achieving more with less: Updated 20th anniversary edition of the productivity and business classic. Hachette, UK.

Lin J (1999). Control of turbulent boundary-layer separation using micro-vortex generators. AIAA Paper, 3404.

Lin J C (2002). Review of research on low-profile vortex generators to control boundary-layer separation, Prog Aerosp Sci, 38:389-420.

Lundstedt T, Seifert E, Abramo L, Thelin B, Nyström A, Pettersen J, Bergman R (1998). Experimental design and optimization. Chemom Intell Lab Syst, 42:3-40.

Mccormick D C (2000). Boundary Layer Separation Control with Directed Synthetic Jets. AIAA Paper, 519.

Montgomery D C (1991). Design and Analysis of Experiments. 3rd ed, John Wiley \& Sons, New York.

Nickerson J D (1986). A study of vortex generators at low reynolds numbers. AIAA Paper, 155.

Pareto V (1964). Cours d'économie politique. vol 1, Librairie Droz.

Prandtl L (1904). Über Flussigkeits bewegung bei sehr kleiner Reibung. III Internat Math Kong, Heidelberg, Teubner, Leipzig, 484-491.

Sado G, Sado M C (2000). Les plans d'expériences : de l'expérimentation à l'assurance qualité. Afnor.

Schlichting H (1979). Boundary-layer theory. 7th ed, McGraw-Hill, New York.

Steele B N, Harding M H (1970). The application of rotating cylinders to ship manoeuvring. National Physical Laboratory.

Taguchi G (1993). Taguchi on Robust Technology Development: Bringing Quality Engineering Upstream. Asme Press Series on International Advances in Design Productivity.

Taylor H D (1947). The elimination of diffuser separation by vortex generators. United Aircraft Corporation Connecticut, Research Department Report No R-4012-3.

Tebbiche H, Boutoudj M S (2015). Passive control on the NACA 4412 airfoil and effects on the lift. Design and Modeling of Mechanical Systems - II (Eds. M. Chouchane et al), Lecture Notes in Mechanical Engineering, Springer, Cham, 775-781.

Thwaites B (1987). Incompressible Aerodynamics: An Account of the Theory and Observation of the Steady Flow of Incompressible Fluid Past Aerofoils, Wings, and Other Bodies. Dover Publication Inc.

Walsh M J (1983). Riblets as a Viscous Drag Reduction Technique. AIAA J, 21:485-486.

Zeng M, Tang L H, Lin M, Wang Q W (2010). Optimization of heat exchangers with vortexgenerator fin by Taguchi method. Appl Therm Eng, 30:1775-1783.

Zhen T K, Zubair M, Ahmad K A (2011). Experimental and numerical investigation of the effects of passive vortex generators on Aludra UAV performance. Chinese J Aeronaut, 24:577-583. 